\title{
A Method to Improve the Effectiveness of Occupational Safety and Health Management System in the Chemical Industry
}

\author{
Hajime Eguchi \\ Consultant, 1-13-9, Mukodai-Cho, Nishitokyo-City, Tokyo-To, 188-0013, Japan \\ h.e@triton.ocn.ne.jp
}

\begin{abstract}
In the chemical industry, Occupational Safety and Health Management System (OSHMS) is introduced to improve the performance of safety for production facilities. However, it is difficult to evaluate the effectiveness of OSHMS. Because the there is no specific performance index in the standards. Whereas, the inner mechanism that encourages the employees' activities for the improvement of the safety and QCD (Quality, Cost, Delivery) with incentives have been introduced positively by the company. Therefore, the current activities in the facility, for example, the result of the existent systems "the near-miss report" and "the proposition for improvement (KAIZEN)". Moreover, as the occurrence of near-miss, accidents or troubles results partially from the error of risk assessment, some penalties should be imposed to the result of risk assessment. In this paper, the index to evaluate the effectiveness of OSHMS, and the practical procedure to utilize the existent systems in the facility and to impose the penalties after the occurrence of near-miss, accidents or troubles are proposed.
\end{abstract}

Keywords: OSHMS, risk assessment, OHSAS18001, chemical industry.

\section{Introduction}

In the chemical industry, OSHMS has been introduced to improve the safety, however, as the evaluation of the effectiveness of OSHMS is hard to do, the propriety of its implementation is often disputed. The Japanese industry has formerly relied upon the personal quality to preserve higher safety and QCD. However, as the plant became complicated and the automatic operation developed, the number of workers has decreased. Meanwhile, the risk in the production process continues to increase. And the measure to reduce the risk has slow down. Therefore, several companies adopt the systems to progress the record of safety and QCD by the incentives to the worker. Nevertheless, these existent trials are insufficient, and many companies have adopted OSHMS in addition to these systems. In the process of OSHMS, the standards require the identification of hazards, risk assessment, planning of risk reduction, performance measurement, monitoring, and checking. However, the procedure to evaluate the effectiveness of risk controls is not indicated clearly. In fact, it is necessary to reflect the fact of near-miss, accidents, and troubles in the facility, as the controls after the risk assessment are insufficient to evaluate the effectiveness of OSHMS accurately. Therefore, in this paper, the index to indicate the degree of total risk reduction and the procedure to reflect the fact of near-miss, accidents and troubles are proposed. These are the procedure to enhance the effectiveness of OSHMS, and to utilize at the management review phase to give the information about the result of risk assessment and the degree of total risk reduction to the management. Now, various standards related to OSHMS are proposed ${ }^{(1-5)}$. However, the future trend of these standards is uncertain, and now the unification of terms is in progress ${ }^{(6)}$. Consequently, as the standard of OSHMS, this paper is based on the standard OHSAS18001 ${ }^{(1)}$.

\section{OHSAS18001 performance measurement and monitoring}

The standard OHSAS18001 has several requirements about the performance measurement and monitoring, and about the effectiveness of controls ${ }^{(1)}$. The objectives of controls are decided at the planning phase and are practiced at the implementation and operation phase. The procedure of risk assessment composes of the hazard identification, risk analysis, and risk controls, if the estimated risk is not 
acceptable, the risk reduction is required. At first, the risk is judged according to the possibility of harms and their consequence, next the risk is classified to the level of risk, and the process of risk reduction is planned as to the level of risk. The standard OHSAS18001 requires the objectives of risk reduction, the measurement and monitoring of the performance of risk reduction. The index is necessary to indicate the difference between the objects and the practiced risk reduction. In fact, if the near-miss, accidents or troubles occur, the risk assessment has to be reviewed. As to the identification of the worker's routine, the hierarchical structure of work in the facility should be considered. Consequently, the procedure to practice the risk assessment about the daily routine of the worker is classified according to the role-based equipment structure by IEC62264 ${ }^{(7)}$.

(a) Identify the daily routine one by one. The start point is the work at a site. And the functional hierarchy of work is classified at an area, at a work center, at a work unit. So that the workers can participate in the risk assessment, the final item of classification is the operation of a work unit.

(b) Segment the operation of a work unit into several actions. Identify the hazard in each action. The hazard is the source of incidence that the hazardous situation occurs through the operation at the work unit, brings to the hazardous event and leads to the harm if it is unavoidable.

The estimation of the level of risk is practiced according to the severity of harm and the frequency of occurrence concerning each hazard. As it is impossible to estimate the frequency of occurrence directly, the frequency of operation is taken into account. The level of risk is classified into three steps according to "as low as reasonably practicable; ALARP" concepts. It contains three regions (unacceptable, tolerable, broadly acceptable). The risk reduction has to be done as to unacceptable to tolerable or broadly acceptable. At the planning phase, the hierarchy of controls is classified as follows: a) elimination, b) substitution, c) engineering controls, d) signage/warning and administrative controls and e) personal protective equipment. According to this hierarchy, the degree of risk reduction is estimated and considered in the planning phase. If an objective of risk reduction is not achieved at the checking phase, this hazard is added again to the objectives at the next planning phase.

\section{Existent systems for improvement of safety}

In the facility, other than OSHMS, various activities are practiced to improve the safety and QCD by the participation of all workers, because in Japan the approach to the safety in the facility has principally been considered to depend on the personal efforts. The representative activities are 1) Nearmiss report system, 2) Proposition for improvement (Kaizen) system. These systems are based on the report and the proposition from workers, and the submitted items from workers are evaluated respectively in the facility and their contributions to the production are evaluated. According to the result of an evaluation, the worker who contributes well receives some incentives from the facility. However, these activities are practiced separately, and there are few opportunity to refer mutually. This section devotes to the activities in the facility other than OSHMS and their concern with OSHMS.

\subsection{Near-miss report}

This system is for the worker to submit the fact of nearmiss that one has met in the daily routine. This report contains not only the situation that one has encountered but also the scheme of the prevention of recurrence. As it concerns with the identification of risk, it is possible to utilize it to the risk assessment in OSHMS. As the risk assessment is mainly based on the assumptive events, its results rarely occurs as the near-miss or accident. The items to correspond to the risk assessment are as follows:

(a) If a risk assessment has already been done, confirm the answering the following questions.

- Is hazard identified?

- Is risk analysis correct?

- Is risk reduction planned and effective?

(b) If a risk assessment has not yet been done, practice the following tasks with the highest priority.

- Execute risk assessment.

- Reflect the near-miss situation to the identification of hazard and the risk analysis.

According to these items, the accuracy and the coverage of risk assessment are improved, as the result of it, the effectiveness of OSHMS becomes higher.

\subsection{Proposition for improvement (Kaizen)}

The proposition for improvement is one of the systems that the workers propose the items to be improved in the daily routine. If the risk assessment is already done, it is necessary to confirm the influence of improvement to the result of risk assessment via the management-of-change 
(MOC) system. If any bad effects exist, the risk assessment must be reviewed before MOC.

(a) If the level of risk goes down,

- The proposition will be accepted.

(b) If the level of risk goes up or other hazards are found,

- If the risk reduction is necessary, it is possible to propose the improvement after an adequate risk reduction.

The result of risk assessment is rarely checked between the intervals of re-assessment. Accordingly it makes frequent opportunity to find some remained errors in the result of the risk assessment by this activity accompanied by the existent systems.

\section{Example of evaluation of effectiveness}

At the evaluation of OSHMS effectiveness, the points of discussion are 1) to establish the index to compare the objectives and the result of risk reduction, 2) to establish the method to apply the facts of near-miss, accident, and trouble to the risk reduction.

\subsection{Index of risk reduction achievement}

The risk of a daily routine in a production process is the object of study in this section. As any standards don't give the performance index, a new index to indicate the achievement of risk reduction is introduced here. The level of risk that is expressed by the matrix form is as follows ${ }^{(8)}$. As to the size of the matrix, several ideas are proposed. In this paper, the size of the matrix is $4 \times 4$ with 5 levels (see Table 1).

Table 1. Level of risk

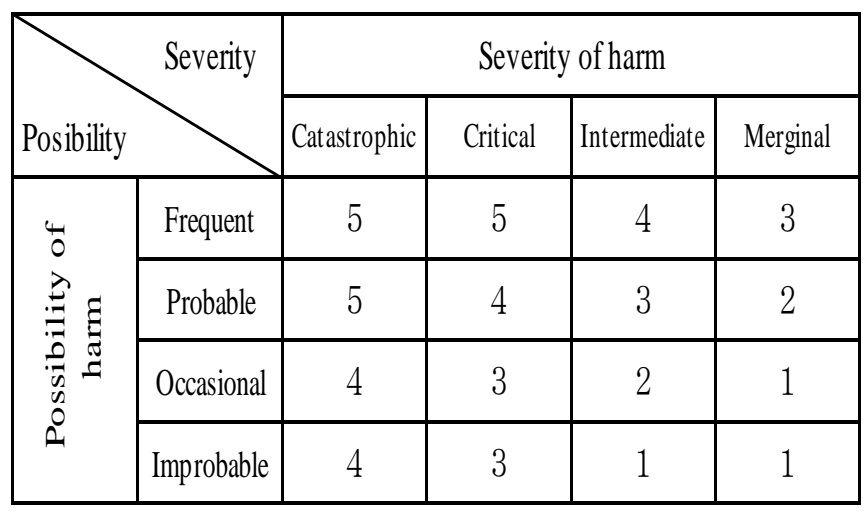

Table2 Hierarchy of risk reduction

\begin{tabular}{|l|l|}
\hline \multicolumn{1}{|c|}{ Priolity of measures } & \multicolumn{1}{c|}{ Specific measures } \\
\hline a) elimination & disuse/change of operation \\
\hline b) substitution & commutation of \\
\hline c) engineering controls & $\begin{array}{l}\text { mechanics of emergency stop, } \\
\text { ventilation }\end{array}$ \\
\hline $\begin{array}{l}\text { d) signage/warning and/or } \\
\text { administrative controls }\end{array}$ & $\begin{array}{l}\text { operation manuals, inhibition } \\
\text { of access, gas detector, alarm } \\
\text { syatem, training }\end{array}$ \\
\hline $\begin{array}{l}\text { e) personal protective } \\
\text { equipment }\end{array}$ & protector \\
\hline
\end{tabular}

d) and e) are adap table to all hazards, but the level of risk is not reduced.

In the concept of ALARP ${ }^{(2)}$, the levels of risk have three regions: unacceptable, tolerable, broadly acceptable. In this paper, the level of risk 5 and 4 are supposed as unacceptable, the level of risk 3 and 2 are tolerable, and the level of risk 1 is broadly acceptable. And in this paper, the level of risk 0 is added as the negligible one. The negligible risk means the disappearance of hazard. In the facility, these levels of risk must be reduced to the tolerable or broadly acceptable region by controls. According to the hierarchy of controls, the degree of risk reduction is fixed (see Table 2). This priority has to be referred at the planning phase to fit into the severity or possibility of harm to prevent the incorrect estimation of risk.

The rule of risk reduction is as follows.

(a) The risk in the unacceptable region must be turned into the tolerable or broadly acceptable region with a), b), c).

(b) The risk in the tolerable region should be turned into the acceptable region with a), b), c).

(c) The risk in the acceptable region should be negligible with a), b) or c).

(d) d), e) should be adapted to all hazards. However the level of risk is not reduced with d), e) except for the hazard that has the level of risk 1. Because d) and e) don't act directly on the hazard. However, in the case of the broadly acceptable risk, d) or e) is a possible measure to reduce the level of risk to the negligible one.

The applicable option to each level is as follows (see Table 3).

At the planning phase, after the selection of the objectives of risk reduction among all hazards by the shift of the level of risk of each hazard, the option to be selected and the degree of risk reduction achievement is fixed. 
Table 3. Option to be selected

\begin{tabular}{c|c|c}
\hline Option & Change of level & $\begin{array}{c}\text { Degree of risk } \\
\text { reduction (level) }\end{array}$ \\
\hline 1 & $5 \rightarrow 0$ & 5 \\
\hline 2 & $5 \rightarrow 1$ & 4 \\
\hline 3 & $5 \rightarrow 2$ & 3 \\
\hline 4 & $5 \rightarrow 3$ & 2 \\
\hline 5 & $4 \rightarrow 0$ & 4 \\
\hline 6 & $4 \rightarrow 1$ & 3 \\
\hline 7 & $4 \rightarrow 2$ & 2 \\
\hline 8 & $4 \rightarrow 3$ & 1 \\
\hline 9 & $3 \rightarrow 0$ & 3 \\
\hline 10 & $3 \rightarrow 1$ & 2 \\
\hline 11 & $2 \rightarrow 0$ & 2 \\
\hline 12 & $1 \rightarrow 0$ & 1 \\
\hline
\end{tabular}

Table 4. Objectives of risk reduction

\begin{tabular}{c|c|c|c|c}
\hline $\begin{array}{c}\text { Level of } \\
\text { risk }\end{array}$ & Option & No. of item & $\begin{array}{c}\text { Change of } \\
\text { level }\end{array}$ & $\begin{array}{c}\text { Degree of risk } \\
\text { reduction (level } \\
\text { per item) }\end{array}$ \\
\hline 5 & 1 & 1 & $5 \rightarrow 0$ & 5 \\
& 4 & 2 & $5 \rightarrow 3$ & 2 \\
\hline 4 & 6 & 2 & $4 \rightarrow 1$ & 3 \\
\hline 3 & 9 & 3 & $3 \rightarrow 0$ & 3 \\
& 10 & 2 & $3 \rightarrow 1$ & 2 \\
\hline 2 & 11 & 5 & $2 \rightarrow 0$ & 2 \\
\hline 1 & 12 & 10 & $1 \rightarrow 0$ & 1 \\
\hline & \multicolumn{3}{|c}{ Risk reduction is incomplete } \\
& \multicolumn{3}{|c}{} \\
\hline
\end{tabular}

\subsection{Analysis of risk reduction by example}

In this section, (a) degree of total risk reduction, and (b) rate of total risk reduction are discussed. In this example, suppose that there are 65 hazards in a production process. And suppose that level 5 contains three items, level 4 contains two items, level 3 contains five items, level 2 contains five items, and level 1 contains 50 items. The objectives of risk reduction at the planning phase is shown in Table 3, and the result of controls at the checking phase is as follows (see Table 4).

(a) Degree of total risk reduction

At the checking phase, if all objectives of risk reduction are completed, the degree of risk reduction is $100 \%$. If three items of level 3 are not completed, the degree of total risk reduction is as follows.

Degree of total risk reduction

$\left.=\sum_{i=1}^{7}\left(\text { Degree per item }_{\mathrm{i}} \times \text { no. of items }\right)_{\mathrm{i}}\right)_{\text {incomplete }}$

$\left./ \sum_{i=1}^{7}\left(\text { Degree per item }_{\mathrm{i}} \times \text { no. of items }\right)_{\mathrm{i}}\right)_{\text {complete }}$

$$
\begin{aligned}
= & (5 \times 1+2 \times 2+3 \times 2+2 \times 2+2 \times 5+1 \times 10) \\
& /(5 \times 1+2 \times 2+3 \times 3+3 \times 2+2 \times 2+2 \times 5+1 \times 10) \\
= & 0.8125
\end{aligned}
$$

(b) Rate of total risk reduction

At the planning phase, the total number of hazards are 100 , and the summation of the total level of risk about total hazards is as follows.

Summation of total level of risk

$=\sum_{i=1}^{5}\left(\right.$ Level of risk $\mathrm{i}_{\mathrm{i}} \times$ no. of items $\left.\mathrm{i}_{\mathrm{i}}\right)$

$=(5 \times 3+4 \times 2+3 \times 5+2 \times 5+1 \times 50)$

$=98$

If three items of level 3 are not completed, the summation of the reduced level of risk is 39 , therefore, the rate of risk reduction is about $40 \%(39 / 98=0.398)$.

At the next planning phase, the number of hazards will reduce from 65 to 44 , because the risk level 0 means the disappearance of hazard.

\subsection{Penalty at near-miss, accidents or troubles}

In the daily routine, the way to cope with the near-miss, accidents or troubles is as follows.

(a) Is the risk assessment performed?

(b) If it is not practiced, it should be practiced immediately. As the risk exists in any action, the identification of hazard and the estimation of risk should be executed referring to the harm of near-miss, accident or trouble.

(c) If it is practiced, the identification of hazard should be checked, i.e. the identification of hazard and the estimation of risk should be reviewed referring to the harm of near-miss, accident or trouble.

(d) At the risk assessment, the identification of hazard and the estimation of risk are almost based on the assumed information; therefore, if the near-miss, accidents or troubles occur, its cause might be an imperfection in the risk assessment. Therefore, some penalty should be imposed to the result of the risk assessment, i.e., the already estimated level of risk has to be raised 1 step.

This procedure is to accelerate the accuracy of the risk assessment. If it is not effective after the controls, this procedure should be repeated continuously.

\section{Conclusion}

OSHMS requires the practice of the planning, the implementation, the operation and the checking phases repeatedly and continuously (PDCA cycle) to measure and to monitor the performance of OSHMS. To activate this 
process, the procedure to show the degree of risk reduction and the rate of risk reduction at the checking phase to inform the achievement of the objectives to the management. And the procedure to impose the penalty on the result of the risk assessment in the case of the occurrence of near-miss, accidents or troubles is proposed. If OSHMS is implemented for the improvement of the safety, the effectiveness should be obvious. However, it is difficult to measure and to monitor it quantitatively. In this paper, the method to improve the reliability of OSHMS is proposed. To practice it effectively, the cooperation of OSHMS with the existent systems in the facility is strongly required. And OSHMS requests us to execute PDCA (Plan-Do-Check-Act) cycle of system repeatedly, and the responsibility to practice this action is in the management in the facility. Consequently these methods will give a lot of suggestion to them.

\section{References}

(1) OHSAS18001: Occupational health and safety management systems - Requirements, 2007
(2) OHSAS18002: Occupational health and safety management systems - Guidelines for the Implementation of OHSAS 18001, 2008

(3) Occupational Health and Safety Management System NOHSC 2002

(4) ISO/DIS 45001.2 Occupational health and safety management systems-Requirements with guidance for use ISO, 2017

(5) ISO31000: 2009 Risk management - Principles and guidelines

(6) ISO GUIDE 73: Risk management - Vocabulary, 2009

(7) IEC62264-1 Enterprise-control system integration Part1: Models and terminology, 2013

(8) IEC61508-5: Functional safety of electrical/electronic/programmable electronic safety related systems - part 5: Examples of methods for the determination of safety integrity levels, 1998

(9) Hajime Eguchi and Donal O’Donovan: “Advancement and Transfer of Skills by Reciprocal Interaction of Human Skills and Techniques via a Production Support System in the Chemical", Journal of Chemical Engineering of Japan, Vol. 43, No. 5, pp. 435-442, 2009 